\section{Carry On (Call Me) Doctor}

\author{
Stephen Hancocks OBE \\ Editor-in-Chief
}

Send your comments to the

Editor-in-Chief,

British Dental Journal,

64 Wimpole Street,

London

W1G 8YS

Email bdj@bda.org
Readers who have a heritage of British comedy film culture will not be slow to conjure up a host of hilarious images and memories based on the famous 'Carry On' movies. A custard pie of a confection somewhere between farce and 'many a true word spoken in jest', they captured something of the essence of the British temperament, its common sense, its humour and its enviable ability to find a sensible compromise; which is why I allude to it in the context of the continuing debate as to whether or not UK qualified dentists should be allowed the courtesy title doctor.

The General Dental Council (GDC) decided at its meeting on 24 February 2011 to delay voting on the issue to allow an 'impact assessment' to take place. The Council had previously given its Standards Committee the task of investigating this, which had a resulted in a recommendation that the Council vote to implement the ban. There were three courses of action that the Council could have taken: to agree with their Committee and no longer permit use of the title, to disagree and throw out the proposal or to seek some middle ground. This latter finds them on the brink but also leaves the door open to some further rational thought on the matter and should be welcomed. Indeed the BDA did just this in a press release issued immediately after the Council meeting had concluded. This also underlined the Association's strongly worded open letter prior to the meeting addressed to the Registrar of the GDC indicating both the potential damaging effect to the dental profession in the UK as a result of such a ban as well as questioning the use of valuable (and expensive) GDC resources in revisiting a subject which has been working perfectly well since 1995.

\section{NO GOOD REASON}

The issue re-emerged, for no good reason of which I am aware, presumably in the GDC's role in protecting the public. Yet as far as the BDA is able to ascertain there has been no evidence of harm or of public complaint in the use of the title. Indeed, the GDC's own research states 'It is important to note that dentists' use of the title "doctor" was not a "top of the mind" issue among respondents'.

I have previously alluded to the debate being akin to that of the heated and exhaustive controversy over fox hunting and I do not particularly want to revisit the whole matter again. However, dentists throughout the world are accorded the title and the plain fact is that the title is just what it says on the tin, a 'courtesy' and a mark of respect. Had the use of the title not been permitted then the debate at this point would have doubtless reached sterility but as it is an accepted and benign actuality to now rescind it would send a far more powerful, demeaning and confusing message to the public than ever continuation of its use would be in danger of doing. One prime example would be the legal ability of a dentist who graduated outside the UK (the EC included) to use a doctorate qualification, while a UK qualified colleague literally in the next door surgery in the same practice (possibly even the practice principal) could not. How does the GDC propose to explain that complexity to a currently non-confused patient?

However, there is one very small chink of light in all of this. The decision to seek further information may indicate that the Council could be starting to recognise that protecting the public can be made more effective if they seek the collaboration of the profession which the previous government disenfranchised rather than demonstrably ignoring, enraging and disaffecting it. The way forward should be very transparent co-operation, to eliminate any accusations of collusion or professional conspiracy, with constructive dialogue on what we all strive to provide; the very best for our patients and the GDC's public. Rather than dissipate valuable resources on the title doctor, the Council might better engage with the profession in educational activities, for example, to continue to improve communication skills, the lack of which are universally agreed to lead to the huge majority of the Fitness to Practise cases under the weight and expense of which the GDC is currently drowning.

What is not in doubt is that we must, if we believe that it is an important aspect of our professional worth, write, email, phone, visit the GDC and do everything at our disposal individually and collectively to communicate the strength of our feeling on the matter. Write. Write now, in advance of their next meeting on 20 May 2011 to consider the issue. We have already let slip far too much of our professional respect and jurisdiction in recent years, potentially to the detriment of our patients as well as ourselves and this is an absolute opportunity that we miss at our peril. In the meantime, just as we have been doing day-in, day-out since 1995 (and before) we will Carry 0n Regardless at what we do best; providing care, treatment and improved oral health.

DOI: 10.1038/sj.bdj.2011.148 